\title{
Parametric Luminescence of Microcavity Polaritons
}

\author{
Cristiano Ciuti ${ }^{1}$, Paolo Schwendimann ${ }^{2}$ and Antonio Quattropani ${ }^{1}$ \\ ${ }^{1}$ Physics Department, Swiss Federal Institute of Technology Lausanne, CH-1015 Lausanne-EPFL, Switzerland \\ ${ }^{2}$ Defense Procurement, System Analysis Division, CH-3003 Bern, Switzerland
}

(July 21, 2018)

\begin{abstract}
The spectral and dispersive emission properties are analytically determined for the twodimensional system of exciton-polaritons in microcavities excited by a resonant and coherent optical pump. New collective excitations result from the anomalous coupling between one generic polariton state and its idler, created by the scattering of two pumped polaritons. The corresponding parametric correlation is stimulated by the emitter and idler populations and drives very efficiently the luminescence. The intrinsic properties of the collective excitations determine a peculiar emission pattern.
\end{abstract}

In their pioneering experiments, Weisbuch et al. [1] discovered a new kind of two-dimensional quasi-particles, resulting from the strong coupling between quantum well excitons and confined photons in a semiconductor microcavity. These peculiar particles, called microcavity polaritons, have a very sharp dispersion due to the very light mass of the cavity photon. Remarkably, this represents a condensed matter system of small mass quasiparticles which can be manipulated through laser beams both in frequency and momentum space. In principle, the low polariton density of states could allow large occupation numbers at relatively small densities of particles, well below the critical saturation value due to the fermionic nonlinearities. In other words, the polariton system could exhibit bosonic properties [2]. Furthermore, unlike unbound electron-hole pairs in ordinary semiconductor lasers, microcavity polaritons have a relatively short time of recombination into external photons. All these ingredients are indeed very promising for applications in the domain of ultrafast all-optical switching and amplification.

Recently, Dang et al. [3] measured photoluminescence spectra from a II-VI microcavity excited with a nonresonant pump. A threshold was observed in the dependence of the polariton luminescence intensity as a function of the input power. Similar results were reported by Senellart and Bloch in a III-V microcavity [ 4 ]. These experiments have been interpreted in terms of enhanced scattering of reservoir excitons into the emitting polariton modes. The origin of the enhancement has been attributed to bosonic stimulation due to final state occupation.

More recently, great insight into the subject has been given by angle-resolved experiments under resonant excitation [5 8]. In this kind of experiments, polaritons are optically excited at a desired energy and momentum, allowing a direct control of the polariton dynamics. In particular, Savvidis et al. [5] have uncovered a new kind of polariton parametric amplifier through pump-probe experiments. The angular selection rules for the para- metric conversion of pumped polaritons into the probe and idler modes are unambiguously given by the energy and momentum conservation for the polariton scattering. In the case of an applied probe, the nonlinearity can be explained in terms of phase-matched wave-mixing of polariton matter beams [9]. Remarkably, giant nonlinearities occur also when the probe beam is absent and the spontaneous emission is detected [5, 6, 8. Spectrally, the luminescence shows a very peculiar distortion with respect to the bare polariton dispersion [6,8]. Moreover, the emission is characterized by a finite angular width which appears to be an intrinsic property of the system [6],8]. Indeed, the whole phenomenology suggests that the interacting polariton matter provides a new kind of collective excitations. The nature of these excitations and the connection with the stimulated scattering mechanism are not yet known. The explicit solution of this intriguing problem is the motivation and main result of our present investigation.

In this paper, we present a theoretical analysis of the polariton nonlinear emission in the case of resonant and coherent pumping. The coherence induced by the pump field allows an anomalous coupling between a generic polariton state and its idler, originated by the fission of two pumped polaritons. The anomalous coupling gives rise to new collective excitations. The luminescence is driven by the emitter-idler parametric correlation which is stimulated by the emitter and idler populations. We establish how the intrinsic properties of the collective excitations determine the rich emission features. In particular, (i) the peculiar dispersion, (ii) the lineshape features and (iii) the two-dimensional pattern are provided by our results.

In order to investigate the nonlinear emission of microcavities in the strong exciton-photon coupling regime, we work directly in the polariton basis. The polariton states have a two-dimensional character and are described by the in-plane wave-vector and their spin. In the following, each $\mathbf{k}$ will be a two-dimensional vector in the quantum well plane. Moreover, we will consider 
the case of a circularly polarized pump beam. Neglecting the spin relaxation, only the polariton states with a definite circular polarization will be retained. When showing numerical results, we will use the angular coordinates $\left(\theta_{x}, \theta_{y}\right)$ defined by $\left(k_{x}, k_{y}\right)=\frac{\omega}{c}\left(\sin \theta_{x}, \sin \theta_{y}\right)$, where $\omega$ is the emission frequency. Actually, these coordinates represent the angles of the far-field images in current experiments [5,6,6,8]. Since we consider the situation of resonant pumping of the lower polariton branch, we can neglect the nonlinear contribution due to the upper branch. The destruction operator for a lower polariton with in-plane wave-vector $\mathbf{k}$ is $p_{\mathbf{k}}=X_{k} b_{\mathbf{k}}+C_{k} a_{\mathbf{k}}$, where $b_{\mathbf{k}}$ and $a_{\mathbf{k}}$ are the exciton and photon Bose operators respectively and $X_{k}, C_{k}$ the corresponding Hopfield coefficients $\left(X_{k}>0\right.$ and $\left.C_{k}<0\right)$. The lower polariton Hamiltonian [9] is $H=H_{L P}+H_{P P}^{e f f}+H_{q m}$. The free term $H_{L P}=\sum_{\mathbf{k}} E_{L P}(k) p_{\mathbf{k}}^{\dagger} p_{\mathbf{k}}$ contains the lower polariton energy dispersion $E_{L P}(k)$. The polariton-polariton interaction term reads

$$
H_{P P}^{e f f}=\frac{1}{2} \sum_{\mathbf{k}, \mathbf{k}^{\prime}, \mathbf{q}} \frac{\lambda_{X}^{2}}{A} V_{\mathbf{k}, \mathbf{k}^{\prime}, \mathbf{q}}^{P P} p_{\mathbf{k}+\mathbf{q}}^{\dagger} p_{\mathbf{k}^{\prime}-\mathbf{q}}^{\dagger} p_{\mathbf{k}} p_{\mathbf{k}^{\prime}},
$$

where $\lambda_{X}$ is the two-dimensional exciton radius and $\mathrm{A}$ is the macroscopic quantization area. The interaction potential is $V_{\mathbf{k}, \mathbf{k}^{\prime}, \mathbf{q}}^{P P}=\left\{2 \frac{\hbar \Omega_{R}}{n_{\text {sat }} \lambda_{X}^{2}}\left(\left|C_{\mathbf{k}+\mathbf{q}}\right| X_{\mathbf{k}^{\prime}}+\left|C_{\mathbf{k}^{\prime}}\right| X_{\mathbf{k}+\mathbf{q}}\right)+\right.$ $\left.\frac{6 e^{2}}{\epsilon \lambda_{X}} X_{\mathbf{k}+\mathbf{q}} X_{\mathbf{k}^{\prime}}\right\} X_{\mathbf{k}^{\prime}-\mathbf{q}} X_{\mathbf{k}}$, with $n_{\text {sat }}=7 /\left(16 \pi \lambda_{X}^{2}\right)$ the exciton saturation density and $\epsilon$ the quantum well dielectric constant. Notice that $V_{\mathbf{k}, \mathbf{k}^{\prime}, \mathbf{q}}^{P P}$ is positive and represents a repulsive interaction. The cavity system interacts with the external electromagnetic field through the standard quasi-mode coupling Hamiltonian $H_{q m}=$ $\int d \Omega\left\{\sum_{\mathbf{k}} g C_{\mathbf{k}} p_{\mathbf{k}}^{\dagger} \alpha_{\mathbf{k}, \Omega}+H . c.\right\}$. The operator $\alpha_{\mathbf{k}, \Omega}$ destructs an external photon with in-plane wave-vector $\mathbf{k}$ and frequency $\Omega$. The spectrum of the polariton luminescence is

$P L(\mathbf{k}, t, \omega) \propto\left|C_{k}\right|^{2} \mathcal{R} e \int_{0}^{+\infty} d \tau e^{-i\left(\omega-i 0^{+}\right) \tau}\left\langle p_{\mathbf{k}}^{\dagger}(t+\tau) p_{\mathbf{k}}(t)\right\rangle$,

where $p_{\mathbf{k}}(t)$ is the time-dependent polariton operator. Here and in the following we use the Heisenberg representation of time-dependent operators and take expectation values on the stationary state. In the steadystate regime, the two-time correlation $\left\langle p_{\mathbf{k}}^{\dagger}(t+\tau) p_{\mathbf{k}}(t)\right\rangle$ depends only on the relative time $\tau$, and not on $t$. This implies that the stationary spectrum can be calculated at a fixed time $t=t_{0}$ when the steady-state regime is achieved. For simplicity, we choose $t_{0}=0$ and hence $P L(\mathbf{k}, \omega) \propto\left|C_{k}\right|^{2} \mathcal{R} e\left\{\left\langle\tilde{p}_{\mathbf{k}}^{\dagger}(\omega) p_{\mathbf{k}}(0)\right\rangle\right\}$, where $\tilde{p}_{\mathbf{k}}(\omega)=\int_{0}^{+\infty} d \tau e^{i\left(\omega+i 0^{+}\right) \tau} p_{\mathbf{k}}(\tau)$.

An applied cw-optical pump with in-plane wavevector $\mathbf{k}_{\mathbf{p}}$ drives a polariton polarization $\left\langle p_{\mathbf{k}_{\mathbf{p}}}(t)\right\rangle=$ $e^{-i \omega_{p} t}\left|\left\langle p_{\mathbf{k}_{\mathbf{p}}}\right\rangle\right|$, where $\omega_{p}$ is the laser frequency resonant with the lower polariton mode. We are interested in the generic polariton operator $p_{\mathbf{k}}(t)$ with $\mathbf{k} \neq \mathbf{k}_{\mathbf{p}}$. The polariton-polariton interaction couples $p_{\mathbf{k}}(t)$ to the pumped mode and to the idler polariton operator $p_{\mathbf{k}_{\text {idler }}}(t)$, where $\mathbf{k}_{\text {idler }}=2 \mathbf{k}_{\mathbf{p}}-\mathbf{k}$. This corresponds to the fission process $\left\{\mathbf{k}_{\mathbf{p}}, \mathbf{k}_{\mathbf{p}}\right\} \rightarrow$ $\left\{\mathbf{k}, \mathbf{k}_{\text {idler }}\right\}$. Disregarding the pump noise, we can perform the replacement $p_{\mathbf{k}}^{\dagger}(t) p_{\mathbf{k}_{\text {idler }}^{\dagger}}^{\dagger}(t) p_{\mathbf{k}_{\mathbf{p}}}(t) p_{\mathbf{k}_{\mathbf{p}}}(t) \simeq$ $p_{\mathbf{k}}^{\dagger}(t) p_{\mathbf{k}_{\text {idler }}}^{\dagger}(t)\left\langle p_{\mathbf{k}_{\mathbf{p}}}(t)\right\rangle^{2}$. Furthermore, we neglect multiple scattering, that is interaction between modes other than the pumped one (i.e. $\left\{\mathbf{k}, \mathbf{k}^{\prime}\right\} \rightarrow\left\{\mathbf{k}+\mathbf{q}, \mathbf{k}^{\prime}-\mathbf{q}\right\}$ ). The limit of validity of this approximation will be discussed later on. With these assumptions, the Heisenberg equations of motion read

$$
\begin{gathered}
i \hbar \frac{d}{d t}\left(\begin{array}{c}
p_{\mathbf{k}}(t) \\
p_{\mathbf{k}_{\mathbf{i d l e r}}}^{\dagger}(t) e^{-i 2 \omega_{p} t}
\end{array}\right)=\hat{M}_{\mathbf{k}}^{p a r}\left(\begin{array}{c}
p_{\mathbf{k}}(t) \\
p_{\mathbf{k}_{\mathbf{i d l e r}}}^{\dagger}(t) e^{-i 2 \omega_{p} t}
\end{array}\right) \\
+\left(\begin{array}{c}
F_{\mathbf{k}}(t) \\
-F_{\mathbf{k}_{\mathbf{i d l e r}}}^{\dagger}(t) e^{-i 2 \omega_{p} t}
\end{array}\right),
\end{gathered}
$$

where the coupling matrix is

$$
\hat{M}_{\mathbf{k}}^{p a r}=\left(\begin{array}{cc}
\tilde{E}_{L P}(\mathbf{k})-i \gamma_{k} & E_{\mathbf{k}}^{i n t} \mathcal{P}_{\mathbf{k}_{\mathbf{p}}}^{2} \\
-E_{\mathbf{k}}^{i n t} \mathcal{P}_{\mathbf{k}_{\mathbf{p}}}^{\star 2} & 2 \hbar \omega_{p}-\tilde{E}_{L P}\left(\mathbf{k}_{\mathbf{i d l e r}}\right)-i \gamma_{k_{\text {idler }}}
\end{array}\right) .
$$

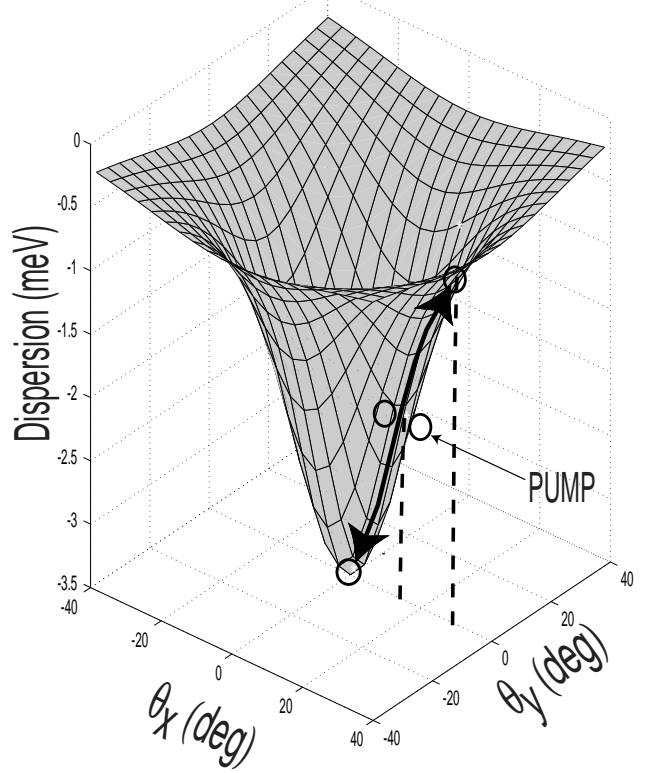

FIG. 1. Lower Polariton energy dispersion $E_{L P}-E_{\text {exc }}(0)$ (meV) versus the angles $\theta_{x}$ and $\theta_{y}$ (deg). A sketch is shown for a parametric fission of two pumped polaritons.

The off-diagonal term contains the energy $E_{\mathbf{k}}^{\text {int }}=$ $\left(V_{\mathbf{k}_{\mathbf{p}}, \mathbf{k}_{\mathbf{p}}, \mathbf{k}-\mathbf{k}_{\mathbf{p}}}^{P P}+V_{\mathbf{k}_{\mathbf{p}}, \mathbf{k}_{\mathbf{p}}, \mathbf{k}_{\mathbf{p}}-\mathbf{k}}^{P P}\right) / 2$ and the pump-induced polarization $\mathcal{P}_{\mathbf{k}_{\mathbf{p}}}=\frac{\lambda_{x}}{\sqrt{A}}\left\langle p_{\mathbf{k}_{\mathbf{p}}}\right\rangle$. We point out that $\left|\mathcal{P}_{\mathbf{k}_{\mathbf{p}}}\right|^{2}=$ $n_{p} \lambda_{X}^{2}$ is the coherent density of pumped polaritons in units of $\lambda_{X}^{-2}$. The diagonal process $\left\{\mathbf{k}, \mathbf{k}_{\mathbf{p}}\right\} \rightarrow\left\{\mathbf{k}, \mathbf{k}_{\mathbf{p}}\right\}$ produces the renormalization of the energy dispersion (blueshift), namely $\tilde{E}_{L P}(\mathbf{k})=E_{L P}(k)+2 V_{\mathbf{k}, \mathbf{k}_{\mathbf{p}}, \mathbf{0}}^{P P}\left|\mathcal{P}_{\mathbf{k}_{\mathbf{p}}}\right|^{2}$. 
Notice the equality of the diagonal elements of $\hat{M}_{\mathbf{k}}^{p a r}$ is equivalent to the exact energy conservation for the process $\left\{\mathbf{k}_{\mathbf{p}}, \mathbf{k}_{\mathbf{p}}\right\} \rightarrow\left\{\mathbf{k}, \mathbf{k}_{\mathbf{i d l e r}}\right\}$. Finally, the coupling to the external photons is responsible for a radiative damping $\gamma_{k}=2 \pi g^{2}\left|C_{k}\right|^{2} / \hbar$ and a Langevin force $F_{\mathbf{k}}(t)=$ $\int d \Omega g C_{\mathbf{k}}^{\star} e^{-i \Omega t} \alpha_{\mathbf{k}, \Omega}(0)$.

From the coupled equations for the operators $p_{\mathbf{k}}(t)$ and $p_{\mathbf{k}}^{\dagger}(t)$, we can obtain the physical quantities of interest. Let us consider the polariton occupation number $N_{\mathbf{k}}(t)=\left\langle p_{\mathbf{k}}^{\dagger}(t) p_{\mathbf{k}}(t)\right\rangle$. From the equations for $p_{\mathbf{k}}(t)$, we get:

$$
\begin{gathered}
\frac{d}{d t} N_{\mathbf{k}}(t)=-\frac{2 \gamma_{k}}{\hbar} N_{\mathbf{k}}(t)+\frac{2}{\hbar} \mathcal{I} m\left\{\left\langle p_{\mathbf{k}}^{\dagger}(t) F_{\mathbf{k}}(t)\right\rangle\right. \\
\left.+E_{\mathbf{k}}^{i n t} \mathcal{P}_{\mathbf{k}_{\mathbf{p}}}^{2} e^{-i 2 \omega_{p} t}\left\langle p_{\mathbf{k}}^{\dagger}(t) p_{\mathbf{k}_{\text {idler }}^{\dagger}}^{\dagger}(t)\right\rangle\right\} .
\end{gathered}
$$

This equation shows that the polariton population $N_{\mathbf{k}}(t)$ is driven by the anomalous quantum correlation $\left\langle p_{\mathbf{k}}^{\dagger}(t) p_{\mathbf{k}_{\text {idler }}}^{\dagger}(t)\right\rangle$. The anomalous correlation evolution reads:

$$
\begin{gathered}
i \hbar \frac{d}{d t}\left\langle p_{\mathbf{k}}^{\dagger}(t) p_{\mathbf{k}_{\text {idler }}}^{\dagger}(t)\right\rangle= \\
{\left[-\tilde{E}_{L P}(\mathbf{k})-\tilde{E}_{L P}\left(\mathbf{k}_{\mathbf{i d l e r}}\right)-i\left(\gamma_{k}+\gamma_{k_{\text {idler }}}\right)\right]\left\langle p_{\mathbf{k}}^{\dagger}(t) p_{\mathbf{k}_{\mathbf{i d l e r}}}^{\dagger}(t)\right\rangle} \\
+\left\langle p_{\mathbf{k}}^{\dagger}(t) F_{\mathbf{k}_{\mathbf{i d l e r}}}^{\dagger}(t)\right\rangle+\left\langle F_{\mathbf{k}}^{\dagger}(t) p_{\mathbf{k}_{\mathbf{i d l e r}}}^{\dagger}(t)\right\rangle \\
\left.-E_{\mathbf{k}}^{i n t} \mathcal{P}_{\mathbf{k}_{\mathbf{p}}}^{\star 2} e^{i 2 \omega_{p} t}\left(1+N_{\mathbf{k}}(t)+N_{\mathbf{k}_{\mathbf{i d l e r}}}(t)\right)\right)
\end{gathered}
$$

Notice that the emitter-idler correlation $\left\langle p_{\mathbf{k}}^{\dagger}(t) p_{\mathbf{k}_{\text {idler }}}^{\dagger}(t)\right\rangle$ has a generation term proportional to the Boson enhancement factor $\left(1+N_{\mathbf{k}}(t)+N_{\mathbf{k}_{\mathbf{i d l e r}}}(t)\right)$. This means that there is a spontaneous inhomogeneous contribution and a stimulation term due to the emitter and idler populations.

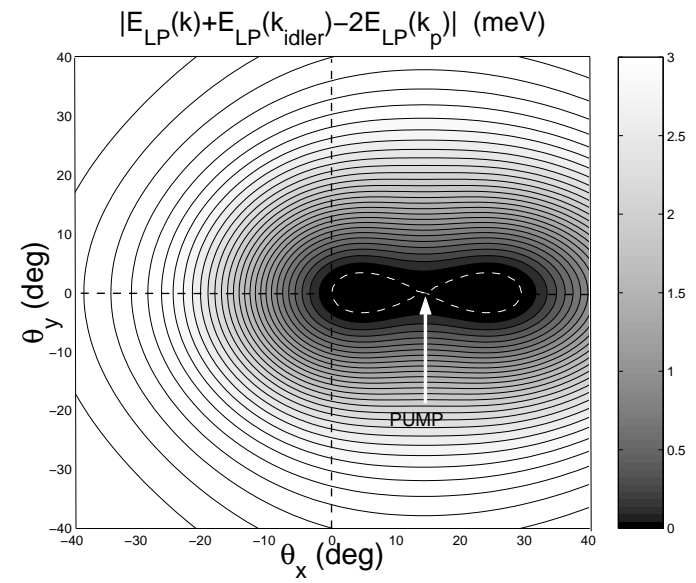

FIG. 2. Contour plot of $\left|E_{L P}(k)+E_{L P}\left(k_{\text {idler }}\right)-2 E_{L P}\left(k_{p}\right)\right|$ $(\mathrm{meV})$ as a function of $\mathbf{k}=\frac{\omega}{c}\left(\sin \theta_{x}, \sin \theta_{y}\right)$.The white dashed line represents the zero value contour, i.e. exact energy-momentum conservation for the process $\left\{\mathbf{k}_{\mathbf{p}}, \mathbf{k}_{\mathbf{p}}\right\} \rightarrow\left\{\mathbf{k}, \mathbf{k}_{\mathbf{i d l e r}}\right\}$. The $\mathrm{x}$-direction is that of the pump wave-vector $\mathbf{k}_{\mathbf{p}}$.
In order to obtain the frequency spectrum of the parametric luminescence, we have to come back to the equations for $p_{\mathbf{k}}(t)$ and $p_{\mathbf{k}}^{\dagger}(t)$. In the frequency space (through the transformation $\left.\int_{0}^{\infty} e^{i\left(\omega+i 0^{+}\right) t} d t\right)$, the coupled equations read

$$
\begin{gathered}
\hbar \omega\left(\begin{array}{c}
\tilde{p}_{\mathbf{k}}(\omega) \\
\tilde{p}_{\mathbf{k}_{\text {idler }}^{\dagger}}^{\dagger}\left(2 \omega_{p}-\omega\right)
\end{array}\right)=\hat{M}_{\mathbf{k}}^{p a r}\left(\begin{array}{c}
\tilde{p}_{\mathbf{k}}(\omega) \\
\tilde{p}_{\mathbf{k}_{\mathbf{i d l e r}}}^{\dagger}\left(2 \omega_{p}-\omega\right)
\end{array}\right) \\
+\left(\begin{array}{c}
\tilde{F}_{\mathbf{k}}(\omega)+i \hbar p_{\mathbf{k}}(t=0) \\
-\tilde{F}_{\mathbf{k}_{\text {idler }}^{\dagger}}^{\dagger}\left(2 \omega_{p}-\omega\right)+i \hbar p_{\mathbf{k}_{\text {idler }}}^{\dagger}(t=0)
\end{array}\right) .
\end{gathered}
$$

Neglecting the noise source $\tilde{F}_{\mathbf{k}}(\omega)$ due to the external photons, the solution of this linear inhomogeneous system is

$$
\tilde{p}_{\mathbf{k}}(\omega)=\frac{\Delta(\omega) i \hbar p_{\mathbf{k}}(t=0)+E_{\mathbf{k}}^{i n t} \mathcal{P}_{\mathbf{k}_{\mathbf{p}}}^{2} i \hbar p_{\mathbf{k}_{\mathbf{i d l e r}}}^{\dagger}(t=0)}{\left(\hbar \omega-E_{+, \mathbf{k}}\right)\left(\hbar \omega-E_{-, \mathbf{k}}\right)}
$$

with $\Delta(\omega)=\hbar \omega+\tilde{E}_{L P}\left(\mathbf{k}_{\mathbf{i d l e r}}\right)+i \gamma_{k_{\text {idler }}}-2 \hbar \omega_{p}$. The complex pole energies $E_{+, \mathbf{k}}$ and $E_{-, \mathbf{k}}$ are the eigenvalues of the parametric matrix $\hat{M}_{\mathbf{k}}^{\text {par }}$ and describe the new collective excitations of the microcavity system. Through the expectation value $\mathcal{R} e\left\{\left\langle\tilde{p}_{\mathbf{k}}^{\dagger}(\omega) p_{\mathbf{k}}(0)\right\rangle\right\}=$ $\mathcal{R} e\left\{\left\langle p_{\mathbf{k}}^{\dagger}(0) \tilde{p}_{\mathbf{k}}(\omega)\right\rangle\right\}$, we obtain the stationary luminescence as a function of the steady state population $N_{\mathbf{k}}^{s}=$ $\left\langle p_{\mathbf{k}}^{\dagger}(0) p_{\mathbf{k}}(0)\right\rangle$ and parametric correlation amplitude $\mathcal{A}_{\mathbf{k}}^{s \star}=$ $\left\langle p_{\mathbf{k}}^{\dagger}(0) p_{\mathbf{k}_{\mathbf{i d l e r}}}^{\dagger}(0)\right\rangle$. The result is

$$
P L(\mathbf{k}, \omega) \propto\left|C_{k}\right|^{2} \mathcal{R} e\left\{i \frac{\Delta(\omega) N_{\mathbf{k}}^{s}+E_{\mathbf{k}}^{i n t} \mathcal{P}_{\mathbf{k}_{\mathbf{p}}}^{2} \mathcal{A}_{\mathbf{k}}^{s \star}}{\left(\hbar \omega-E_{+, \mathbf{k}}\right)\left(\hbar \omega-E_{-, \mathbf{k}}\right)}\right\} .
$$

Simple algebra shows that steady-state solutions of the coupled equations for population and parametric correlation are $\left\langle p_{\mathbf{k}}(t) p_{\mathbf{k}_{\mathbf{i d l e r}}}(t)\right\rangle=\mathcal{A}_{\mathbf{k}}^{s} e^{-i 2 \omega_{p} t}$ and $N_{\mathbf{k}}(t)=N_{\mathbf{k}}^{s}$. The anomalous correlation amplitude reads

$$
\mathcal{A}_{\mathbf{k}}^{s}=\frac{E_{\mathbf{k}}^{i n t} \mathcal{P}_{\mathbf{k}_{\mathbf{p}}}^{2} \delta_{\mathbf{k}}}{\left|\delta_{\mathbf{k}}\right|^{2}-\frac{\left(\gamma_{k}+\gamma_{k_{\text {idler }}}\right)^{2}}{\gamma_{k} \gamma_{\text {idler }}}\left|E_{\mathbf{k}}^{i n t} \mathcal{P}_{\mathbf{k}_{\mathbf{p}}}^{2}\right|^{2}},
$$

with $\delta_{\mathbf{k}}=\left(2 \hbar \omega_{p}-\tilde{E}_{L P}(\mathbf{k})-\tilde{E}_{L P}\left(\mathbf{k}_{\mathbf{i d l e r}}\right)-i\left(\gamma_{k}+\gamma_{k_{\text {idler }}}\right)\right)$. Moreover, the steady-state population is

$$
N_{\mathbf{k}}^{s}=\frac{1}{\gamma_{k}} \mathcal{I} m\left(E_{\mathbf{k}}^{i n t} \mathcal{P}_{\mathbf{k}_{\mathbf{p}}}^{2} \mathcal{A}_{\mathbf{k}}^{s \star}\right)
$$

Of course, $N_{\mathbf{k}_{\text {idler }}}^{s}=N_{\mathbf{k}}^{s} \gamma_{k} / \gamma_{k_{\text {idler }}}$. As we will show below, the PL signal (as well as the parametric correlation amplitude $\mathcal{A}_{\mathbf{k}}^{s}$ and population $N_{\mathbf{k}}^{s}$ ) diverges when the pump density $\left|\mathcal{P}_{\mathbf{k}_{\mathbf{p}}}\right|^{2}$ reaches a threshold value. In fact, above threshold, the pump polarization can be no longer treated 
as a parameter. This means that the equation of motion for the pumped mode has to be included, accounting for the pump depletion. Moreover, when the transfer from the pump wave-vector to the other ones becomes very massive, multiple scattering can be no longer neglected. Hence, the results here presented are valid only below the threshold of the parametric luminescence.
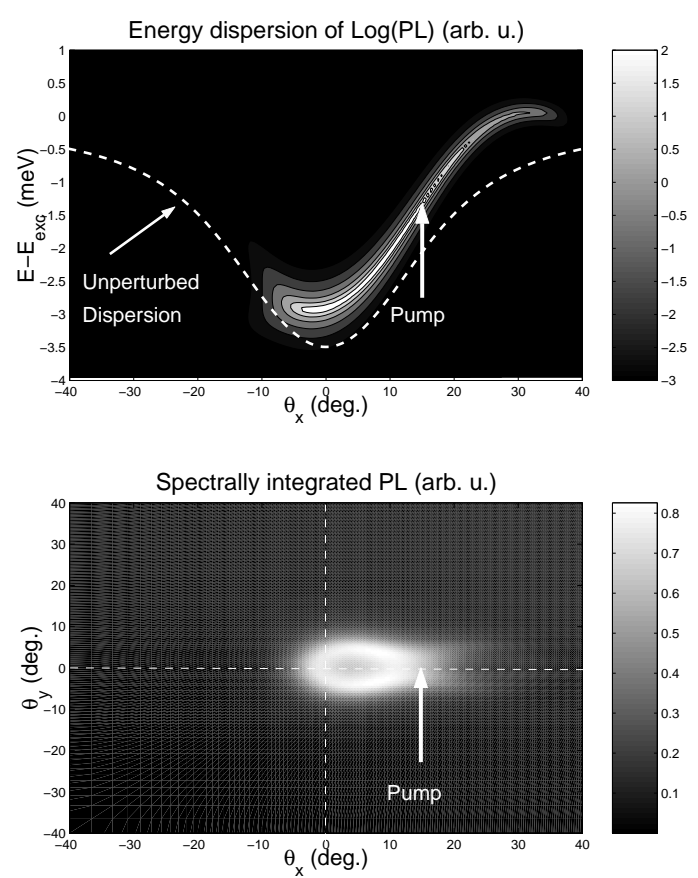

FIG. 3. (a)Contour plot of the parametric photoluminescence (log scale) as a function of the angle $\theta_{x}$ (deg) and energy (meV) for the pumped density $n_{p}=0.03 n_{\text {sat }}$ and $\theta_{y}=0$. The dashed-line is the unperturbed dispersion of the lower polariton branch. (b) Two-dimensional pattern of the spectrally integrated emission.

For sake of illustration, we present our analytical results by using GaAs parameters for a realistic microcavity structure with Rabi splitting equal to $7 \mathrm{meV}$. We consider the situation of zero exciton-photon detuning, i.e. $E_{\text {exc }}(0)=E_{c a v}(0)$. For simplicity, we consider a k-independent polariton linewidth $\gamma_{k} \approx 0.4 \mathrm{meV}$ (for large $\mathrm{k}$, the decreasing of the radiative width is usually compensated by nonradiative broadening). The twodimensional dispersion of the lower polariton branch is shown in Fig. 1. We consider a pump which resonantly excites the polariton branch at the critical wave-vector such as $E_{L P}(0)+E_{L P}\left(2 k_{p}\right)=2 E_{L P}\left(k_{p}\right)$, allowing the particular polariton fission $\left\{\mathbf{k}_{\mathbf{p}}, \mathbf{k}_{\mathbf{p}}\right\} \rightarrow\left\{\mathbf{0}, \mathbf{2} \mathbf{k}_{\mathbf{p}}\right\}$ (with increasing pumping, one has to consider the renormalized dispersion $\tilde{E}_{L P}$ instead of $\left.E_{L P}\right)$. Fig. 2 contains the contour plot of the quantity $\mid E_{L P}(\mathbf{k})+E_{L P}\left(\mathbf{k}_{\mathbf{i d l e r}}\right)-$ $2 E_{L P}\left(\mathbf{k}_{\mathbf{p}}\right) \mid$ in $\mathbf{k}$ space $\left(\mathbf{k}_{\mathbf{p}}\right.$ is along the $\mathrm{x}$-direction). The white-dashed line represents the zero value contour, i.e. exact energy conservation for the fission $\left\{\mathbf{k}_{\mathbf{p}}, \mathbf{k}_{\mathbf{p}}\right\} \rightarrow$ $\left\{\mathbf{k}, \mathbf{k}_{\mathbf{i d l e r}}\right\}$. Remarkably, the conservation is only weakly forbidden in a large squeezed portion of $\mathbf{k}$-space (dark region) around $\mathbf{k}_{\mathbf{p}}$. This implies that the parametric coupling is efficient on a broad angular range. This is actually found in Fig.3(a), where a typical contour plot of the photoluminescence (log scale) is shown as a function of the angle $\theta_{x}\left(\theta_{y}=0\right)$ and of the emission energy. Moreover, Fig. 3(b) shows the two-dimensional pattern of the spectrally integrated emission (linear scale). The depression of the emission at large angles is due to the vanishing cavity fraction $\left|C_{\mathbf{k}}\right|^{2}$.
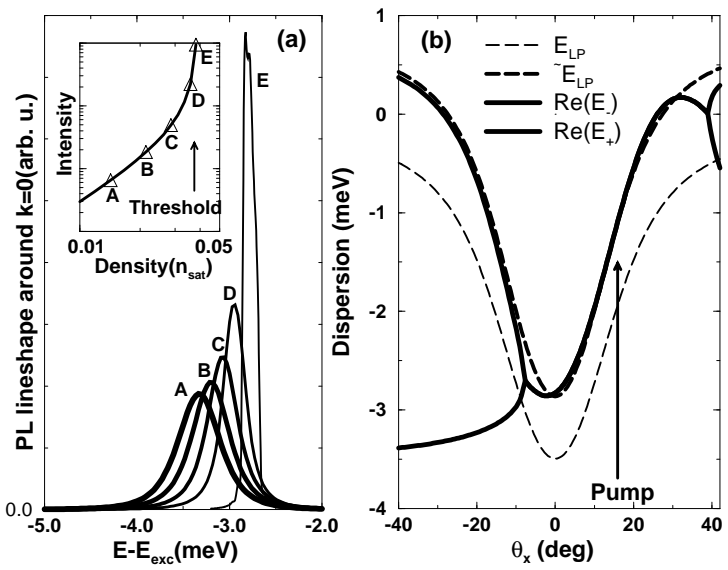

FIG. 4. (a) Normalized emission lineshape around $\mathrm{k}=0$ $\left(\theta_{x}=\theta_{y}=0\right.$; angular acceptance of $\left.2 \mathrm{deg}\right)$ for 5 densities (A-E). Inset: log-log plot of the emission intensity versus pumped density ( $n_{\text {sat }}$ units). (b) Dispersions (meV) versus $\theta_{x}$ (deg) for $\theta_{y}=0$ at the highest pump density. Thin dashed line: unperturbed polariton dispersion $E_{L P}$. Thick dashed line: blueshifted dispersion $\tilde{E}_{L P}$. Thick solid line: $\mathcal{R} e\left(E_{ \pm}\right)$ with $\mathcal{R} e\left(E_{-}\right) \leq \mathcal{R} e\left(E_{+}\right)$. The bifurcation occurs when the violation of the energy conservation is large enough and implies negligible parametric emission.

To determine the dependence on the pump intensity, we just need to analyze the evolution of the eigenvalues $E_{ \pm, \mathbf{k}}$. For a given $\mathbf{k}$ satisfying exactly the energymomentum conservation for the parametric scattering, the expression for the pole energies $E_{ \pm, \mathbf{k}}$ is very simple, i.e. $\mathcal{R} e\left(E_{ \pm, \mathbf{k}}\right)=\tilde{E}_{L P}(\mathbf{k})$ and $\left|\mathcal{I} m\left(E_{ \pm, \mathbf{k}}\right)\right|=$ $\left(\sqrt{\gamma_{k} \gamma_{k_{\text {idler }}}} \pm E_{\mathbf{k}}^{\text {int }}\left|\mathcal{P}_{\mathbf{k}_{\mathbf{p}}}\right|^{2}\right)$. This means that the imaginary part of the pole energy $E_{-, \mathbf{k}}$ gets smaller at the expense of the other pole $E_{+, \mathbf{k}}$. Consequently, the photoluminescence spectrum $P L(\mathbf{k}, \omega)$ gets spectrally narrower. The threshold density for the parametric luminescence is defined by $\operatorname{Im}\left(E_{-, \mathbf{k}}\right)=0$, i.e. $E_{\text {int }}\left|\mathcal{P}_{\mathbf{k}_{\mathbf{p}}}\right|^{2}=\sqrt{\gamma_{k} \gamma_{k_{\text {idler }}}}$. For this value, the PL signal, the parametric correlation amplitude $\mathcal{A}_{\mathbf{k}}^{s}$ and steady-state population $N_{\mathbf{k}}^{s}$ diverge and therefore the pump depletion has to be included. All these features can be seen explicitly in Fig. 4. With increasing pump density the lineshape of the emission around $k=0$ blueshifts and narrows (Fig. 4(a)). The output intensity (see the log-log plot inset) shows a threshold behavior as a function of the coherent density of pumped polaritons $n_{p}=\left|\mathcal{P}_{\mathbf{k}_{\mathbf{p}}}\right|^{2} / \lambda_{X}^{2}$. Notice that when the polariton damping $\gamma_{k}$ is small enough, the threshold density $n_{t h r}$ can be orders of magnitude smaller than the 
exciton saturation density $n_{\text {sat }}$. With the realistic material parameters employed here, we have $n_{t h r} \approx 0.05 n_{\text {sat }}$. Finally, Fig.4(b), shows explicitly the energy dispersions of the collective excitations. When the energy conservation for the parametric fission is well satisfied, $\mathcal{R} e\left(E_{ \pm}(\mathbf{k})\right) \simeq \tilde{E}_{L P}(\mathbf{k})$. On the other hand, when the energy conservation is strongly broken and consequently the parametric luminescence is negligible, $\mathcal{R} e\left(E_{-}(\mathbf{k})\right)$ and $\mathcal{R} e\left(E_{+}(\mathbf{k})\right)$ bifurcate, tending to the diagonal elements of the matrix $M_{\mathbf{k}}^{\text {par }}$, i.e. $\tilde{E}_{L P}(\mathbf{k})$ and $2 \hbar \omega_{p}-\tilde{E}_{L P}\left(\mathbf{k}_{\mathbf{i d l e r}}\right)$. In the intermediate case, consistently with experiments, the dispersion $\mathcal{R} e\left(E_{ \pm}(\mathbf{k})\right)$ shows peculiar features such a change of sign of the in-plane group velocity [8] around $\mathbf{2} \mathbf{k}_{\mathbf{p}}$ and a flattening [6, 8 around $\mathbf{k}=\mathbf{0}$ (see also the emission spectra in Fig. 3).

In conclusion, we have theoretically described the parametric luminescence of microcavity polaritons, showing the subtle interplay between parametric correlation and stimulated scattering. The new collective excitations resulting from the anomalous emitter-idler coupling produce a very peculiar luminescence pattern in frequency and momentum space, giving a key to recent experiments [5, 6, 8]. Further investigations are in progress for the regime above threshold not treated in this paper, but investigated in new experiments [10]. We wish to thank J.J. Baumberg, B. Deveaud, R. Houdré, M. Saba, P.G. Savvidis, R.P. Stanley, F. Tassone and C. Weisbuch for fruitful discussions.

[1] C. Weisbuch et al., Phys. Rev. Lett. 69, 3314 (1992).

[2] E. Hanamura and H. Haug, Phys. Rep., Phys. Lett. C 33, 209 (1977).

[3] Le Si Dang et al., Phys. Rev. Lett. 81, 3920 (1998).

[4] P. Senellart and J. Bloch, Phys. Rev. Lett. 82, 1233 (1999).

[5] P.G. Savvidis et al., Phys. Rev. Lett. 84, 1547 (2000).

[6] R. Houdré et al., Phys. Rev. Lett.85, 2793 (2000).

[7] R. Huang, F. Tassone and Y. Yamamoto, Phys. Rev. B 61, R7854 (2000).

[8] P.G. Savvidis et al., Phys. Rev. B 62, RXXXX (2000).

[9] C. Ciuti et al., Phys. Rev. B 62, R4825 (2000).

[10] J.J. Baumberg et al., Phys. Rev. B 62, RXXXX (2000); R.M. Stevenson et al., Phys. Rev. Lett. 85, 3680 (2000). 